\title{
A practical approach to diagnostic Ig/TCR clonality evaluation in clinical pathology
}

\author{
Patricia J. T. A. Groenen - Annemiek van Raaij • \\ Margarethe C. van Altena • Paul M. Rombout • \\ J. M. Han van Krieken
}

Received: 14 August 2011 / Accepted: 12 December 2011 /Published online: 11 January 2012

(C) Springer-Verlag 2012

\begin{abstract}
The BIOMED-2 group developed and approved a set of multiplex Ig and T cell receptor PCR primers and successfully applied these to different well-defined WHO lymphoma entities with unprecedented high frequencies of malignant cases showing clonality. This approach has now become a worldwide standard of clonality testing in lymphoproliferations. While the clonality testing and assessment by GeneScan and/or heteroduplex analysis has become relatively easy to perform, the evaluation of the obtained gene rearrangement patterns can be difficult. In this review, we will address specific aspects of clonality testing, concerning both the practical phase as the evaluation of the obtained gene rearrangement patterns, which will help to overcome problems that can be encountered in the routine diagnostic setting.
\end{abstract}

Keywords Clonality pattern evaluation $\cdot \operatorname{Ig} / \mathrm{TCR}$ clonality interpretation · GeneScan · Aberrant TCRG-JJ rearrangement

\section{Introduction}

Clonality assessment is an important supplementary tool for pathologists who encounter a lymphoproliferative lesion that is difficult to interpret. The EuroClonality/ BIOMED-2 group developed primer sets to detect clonal antigen receptor gene rearrangements [1] and protocols to use them $[2,3]$. This methodology results in clonality detection in virtually all $\mathrm{B}$ and $\mathrm{T}$ cell lymphoma cases $[4,5]$. Clonality assessment is useful in the cases where

P. J. T. A. Groenen $(\bowtie) \cdot$ A. van Raaij • M. C. van Altena

P. M. Rombout · J. M. H. van Krieken

Department of Pathology,

Radboud University Nijmegen Medical Centre, PO Box 9101, 6500 HB Nijmegen, The Netherlands e-mail: P.Groenen@pathol.umcn.nl the discrimination between malignancy and reactive is difficult, in cases of suspect $\mathrm{B}$ or $\mathrm{T}$ cell proliferations at specific sites or in the context of immunodeficiency and in Bcl2-negative follicular lymphomas [6]. Clonality assessment is thus an important tool in the diagnosis of malignant lymphoproliferations, even though clonality does not always imply malignancy, as some reactive processes contain large clonal lymphocyte populations [7]. Clonality detection is, however, not suitable to discriminate between Hodgkin's and non-Hodgkin's lymphoma, as the BIOMED-2 methods are sufficiently sensitive to detect small numbers of malignant cells in many cases of classical Hodgkin's lymphoma [8-11].

The majority of B cell neoplasms has clonal $I G H(V D J)$ and $I G K$ gene rearrangements with clear complementarity of clonality detection [4], which is a one of the advantages of the BIOMED-2 clonality testing approach. Furthermore, the detection of both a clonal $I G H$ and $I G K$ rearrangement in itself is a confirmation of clonality. Likewise, the majority of T cell neoplasms have clonal $T C R G$ and TCRB gene rearrangements [5].

Multiplex PCR-based clonality testing and assessment by GeneScan and/or heteroduplex analysis has become a worldwide standard [12-18]. Technically, molecular clonality testing and assessment by GeneScan and/or heteroduplex analysis has become relatively easy to perform. However, by the discussion of the cases presented in the EuroClonality workshops: "Clonality assessment in pathology" (website: http://www.euroclon ality.org), it has become clear that inclusion of quality checks in the routine diagnostic setting, knowledge of the gene loci and experience with $\mathrm{Ig} / \mathrm{T}$ cell receptor (TCR) rearrangement analysis are essential to avoid misinterpretation of the data. In this review, we will share experiences of the workshops, discuss pitfalls and provide recommendations for practical clonality testing that will facilitate unequivocal evaluation of gene rearrangement patterns of cases in clinical pathology. 
The importance of PCR duplicates and sufficient DNA input in clonality testing

The evaluation of clonality patterns is not a sinecure especially in cases that are difficult for the pathologist. It can be hard to discern whether the entire pattern of rearrangements is truly clonal, oligoclonal, polyclonal or pseudoclonal. In our experience, duplicate assessment in clonality testing is helpful and in fact essential, not only to confirm clonality (Fig. 1a, b) but also to prevent over-interpretation. Figure 1c shows a slightly dominant signal in a background of polyclonal Gaussian curve, which might mistakenly be interpreted as "clonal". However, the second PCR result from the duplicate experiment clearly shows true polyclonality (Fig. 1c). In this respect, running duplicates facilitates interpretation. Duplicate assessment is also helpful for the evaluation of cases that do not display perfectly shaped Gaussian curves, as shown in Fig. 1d. These PCR assessments reveal "spiked" peaks on top of a Gaussian curve; however, on the whole, the pattern clearly is polyclonal.

For duplicate assessment, we recommend to use $100 \mathrm{ng}$ DNA per $50 \mu 1$ PCR mix as input per PCR for DNA samples obtained from snap-frozen tissues and 50 and $200 \mathrm{ng}$ of affinity-purified (not a rough DNA concentrate) DNA per PCR $(50 \mu \mathrm{l})$ for formalin-fixed and paraffin-embedded (FFPE) samples. The two different DNA concentrations are being used as "duplicates" in the analysis because of the probability of PCR-inhibiting factors in the DNA sample.

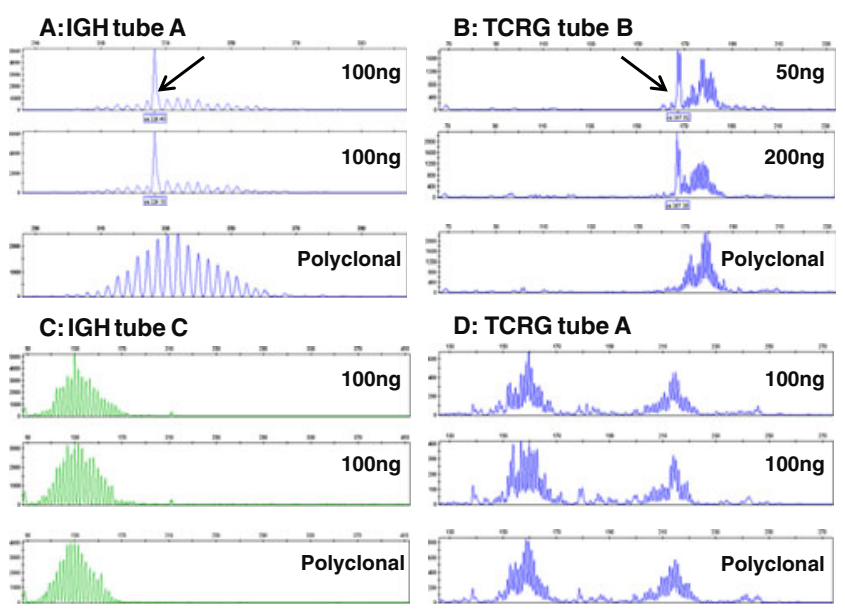

Fig. $1 \mathrm{Ig}$ and TCR gene rearrangement patterns showing the added value of duplicate assessment. In each panel, duplicates of the PCRs of the sample are shown and a polyclonal control of the target is included. a Clonal $I G H$-VDJ rearrangement within hardly any polyclonal background, b clonal TCRG-VJ in polyclonal background. Polyclonal $I G H$ VDJ and $T C R G-\mathrm{VJ}$ rearrangements are shown in $\mathbf{c}$ and $\mathbf{d}$, respectively. Duplicate assessment is helpful to confirm clonality $(\mathbf{a}, \mathbf{b})$ and avoid over-interpretation of a seemingly clonal peak (upper pattern c) or "spiked" Gaussian curves (d). a, c and d From DNA obtained from fresh frozen tumour samples, b from DNA from a formalin-fixed and paraffin-embedded tumour sample. The arrow indicates the clonal rearrangement
Duplicate assessment and sufficient DNA input are important to exclude pseudo-clonality, a phenomenon that may occur in case of low input of target B or T lymphocytes. Figure 2a illustrates a diagnostic request that, upon routine analysis, resulted in pseudoclonality, i.e. seemingly "clonal" peaks of different amplicon lengths in the gene rearrangement patterns of the replicates (two upper panels). In a single experiment, a dominant peak, like the one observed in this case, might be falsely interpreted as clonal. However, by adjusting the DNA input from the tumour sample to $300 \mathrm{ng}$, the evaluation of the case becomes clear, as a polyclonal rearrangement pattern can be observed (Fig. 2a, two lower panels).

A single dominant peak of low signal intensity, but reproducibly detected (Fig. 2b), may represent a true rearrangement. Caution should be taken upon interpretation of these patterns, and confirmation is needed. In this particular case, sequence analysis of this dominant peak enabled us to demonstrate a true $I G K$-DE rearrangement, thereby confirming a clonal result and excluding mispriming of the primers.

Tissue issues in clonality analysis

DNA quality, which determines efficiency of DNA amplification, is an important parameter for clonality analysis as well, especially for DNA samples from FFPE samples. Although the BIOMED-2 Ig and TCR clonality PCRs were designed and tested on DNA samples obtained from fresh tissues, our experience is that the primers work well on DNA's obtained from the majority of FFPE tissues. Critical factors that determine the outcome of the clonality assessment using FFPE are the fixation type and fixation time and

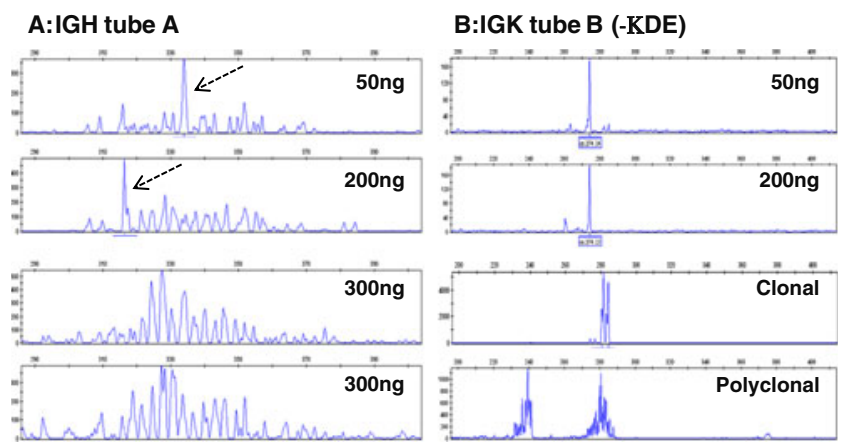

Fig. 2 Gene rearrangement patterns of low intensity signals: the relevance of duplicates. a: $I G H$-FR1 (tube A) replicates that show low intensity signals with differently sized "pseudoclonal peaks" (as indicated by the dashed arrows) upon DNA input of 50 and 200 ng per PCR. Upon increase in the DNA input to $300 \mathrm{ng}$ per PCR, this case displays a polyclonal (irregular) pattern of rearrangements. DNA concentrations were measured by the Nanodrop 2000 (Thermo Fisher Scientific, Wilmington, DE, USA, www.nanodrop.com). b IGK-DE (tube B) replicates that demonstrate reproducible but low intensity clonal signals. By sequence analysis, this clonal peak was confirmed to represent a true clonal $I G K-\mathrm{DE}$ gene rearrangement 
tissue processing $[19,20]$. The usage of buffered formalin and short fixation times will ensure sufficient DNA quality, which allows PCR amplification in the size range of around 200-300 bps, which is the size range the majority of the BIOMED-2 PCR amplicons. A number of the BIOMED-2 PCRs, however, yield amplicon sizes of around $300 \mathrm{bp}$ or even more, which indeed may be difficult targets that may go undetected when working with suboptimal DNA samples. From the TCR targets, the TCRB tube C PCR for the incomplete rearrangements $(D \beta-J \beta)$ yield high amplicon sizes of around $300 \mathrm{bp}$ in size, for especially the $\mathrm{D} \beta 1-\mathrm{J} \beta$ rearrangements. However, since the other TCR PCR targets have smaller amplicon sizes, the multi-target approach combined with the complementarity of the targets is informative in the majority of cases that enable amplification of the $200 \mathrm{bp}$ gene control PCR. Besides, the occurrence of an isolated $\mathrm{D} \beta 1-\mathrm{J} \beta$ rearrangement is very rare [5]. Suboptimal DNA samples that enable PCR amplification of only the $100 \mathrm{bp}$ band of the control gene PCR [1] cannot be properly evaluated for $\mathrm{T}$ cell clonality in our opinion.

For the Ig gene targets, the $I G H$-FR1 PCR yield amplicon sizes of above $300 \mathrm{bp}$, and also the $\mathrm{Vk} 2 / \mathrm{VK} 4 / \mathrm{Vk} 5$ gene rearrangements yield amplicon sizes of around $300 \mathrm{bp}\left(-\mathrm{JK}_{\mathrm{K}}\right.$ rearrangements) or even 350-390 bp (KDE rearrangements). Interestingly, we and others [21] observed that $I G K$ is a very good target with a high detection rate of clonality, even in cases of suboptimal DNA quality. An example is provided in Fig. 3. Similar to the TCR gene assessment, the multi-target approach has added value for the assessment of the Ig targets for B cell lymphoma diagnostic requests. For B cell lymphoma requests, suboptimal DNA samples that enable PCR amplification of only the $100 \mathrm{bp}$ band of the control gene PCR might provide information for the $I G H$ (FR3) and/or $I G K$-PCRs in case a convincing, reproducible clonal product is detected. More issues on the use of FFPE tissues and clonality assessment will be described by Lenze et al. [20].

Next to lymph nodes, molecular assessment of a bone marrow trephine biopsy is relevant in the diagnostic workup of patients with haematological disorders. Fixation of bone marrow biopsies in buffered formalin followed by decalcification in neutral EDTA renders good DNA preservation [22]. The storage of the formalin-fixed decalcified paraffin-embedded trephine blocks (up to 200 months) had no impact on the ability to amplify specimens [23]. By the use of such protocols, the majority of our in-house bone marrow trephines allows successful B and/or T cell clonality assessment. Unfortunately, fixation and decalcification procedures for bone marrow biopsies are not standardised throughout the different countries or even laboratories, potentially causing failures in clonality assessment particularly in those cases that are obtained using acid-based reagents for decalcification. As an alternative, archival-stained bone

\section{IGK tube $B(-K D E)$}
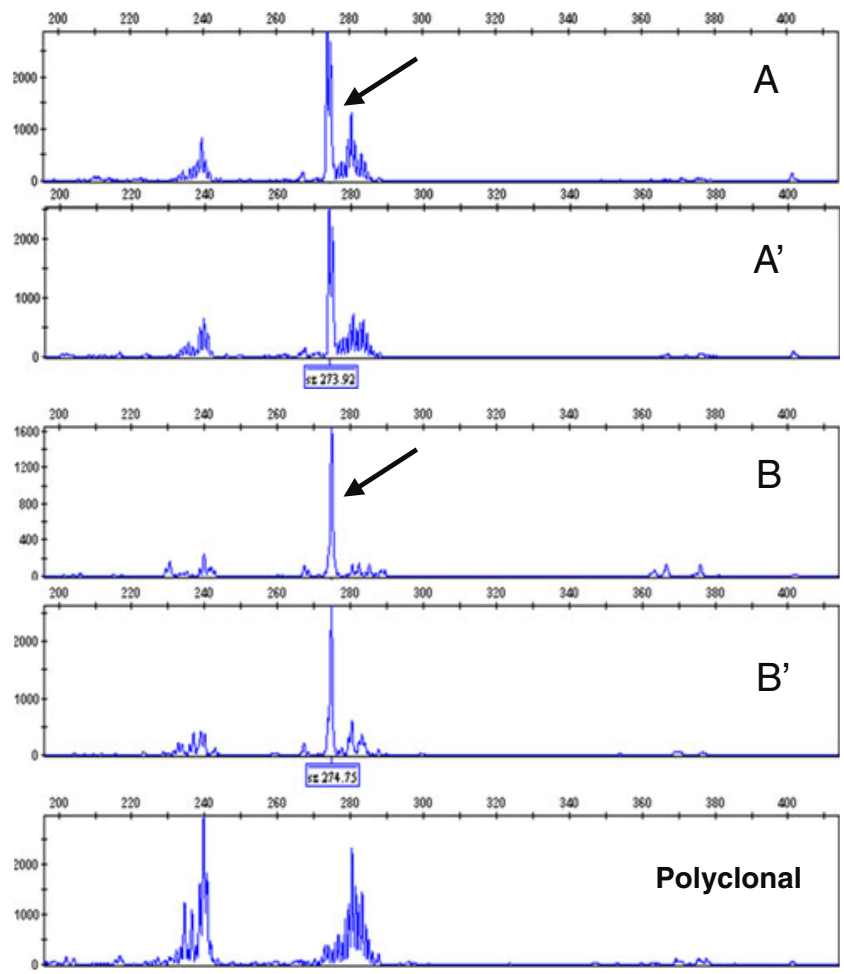

Fig. 3 Clonal $I G K$-DE rearrangements can be easily detected in DNA of suboptimal quality. Gene rearrangement patterns $\mathrm{A} / \mathrm{A}^{\prime}$ (duplicates) were obtained from DNA from fresh frozen specimen yielding optimal DNA quality. The patterns B/B' (duplicates) were obtained from DNA from the corresponding FFPE sample that allowed amplification of only a weak $200 \mathrm{bp}$ band as highest product in the gene control PCR. The clonal IGK-DE product of 274 bp was easily detectable in the suboptimal DNA sample

marrow slides can be used. Cells can be scraped-off from archival Giemsa-stained bone marrow smears or from cytological smears and can be used for DNA extraction [24]. In general, DNA samples from Giemsa-stained smears provide optimal DNA quality and allow amplification of 300-600 bp band from the control gene PCR. Similar to the histological cases, also in the cytological cases, it is essential to have checked the representativity in terms of presence of (malignant) lymphocytes to ensure reliable clonality testing.

In cases with a low percentage of suspicious malignant B or T lymphocytes, adjustment of the amount of DNA by increasing the DNA concentration and hence the number cells per PCR can be useful. This approach may help in the assessment of clonality in skin biopsies, which can be problematic because these biopsies often contain quite scanty lymphoid populations in combination with a high proportion of keratin. The low number of lymphocytes can result in over-interpretation of incidentally dominant peaks. Also in these cases, it is important to run duplicate reactions, to increase the amount of DNA analysed and to assess multiple biopsies from different skin sites, when these are available, 
to look for consistently occurring peaks $[25,26]$. It may also be helpful to run the products on a heteroduplex gel, which can overcome the problem of over-interpretation of incidentally dominant peaks. When performed appropriately, PCRbased TCRG and TCRB clonality tests can be used in the differential diagnosis of mycosis fungoides and reactive inflammatory dermatoses, which are difficult to distinguish clinically and histopathologically [27].

Interpretation of clonality: not a strictly quantitative approach

Although a range of DNA concentrations will generate a valid result, using sufficient DNA per PCR target as input and adjusting DNA input in cases of low tumour load is essential in clonality assessment and cannot be stressed enough. We are aware that certain commercial kits recommend criteria for defining a positive peak, meaning that true positive clonal products should fall within the valid size range and should be at least three times the amplitude of the third largest peak in the polyclonal background. In our opinion, it is not correct to determine a ratio, simply because the polymerase chain reaction is not quantitative. In addition, short and long junctional regions more likely lead to a conclusion of "clonal", compared to intermediately sized junctional regions. Thus, the size of the junctional region influences a strictly quantitative approach. Furthermore, the observed ratio is strongly affected by the DNA input, as is shown in Fig. 4a and b. In clear clonal cases without background signal, DNA input hardly affects the pattern (Fig. 4c).

In fact, the DNA input implicitly represents the tumour load, e.g. the percentage of suspect malignant lymphocytes that are in the tissue. We therefore recommend usage of the histopathological information (or the information from flow cytometry) for estimation of the percentage of suspect malignant and normal (reactive) $\mathrm{B}$ and $\mathrm{T}$ cells and integrate these data with the molecular clonality patterns to provide the most reliable diagnosis. For example, a case that shows reproducible (small) clonal results in a polyclonal background, in multiple PCR targets, which fit in the context of the histological information, undoubtedly is consistent with the presence of a small clonal population (see [3], for additional examples]. These minor B or $\mathrm{T}$ cell clones are not necessarily malignant but may arise in the context of the disease, which is why a good correlation with histology and the clinical information is important. Tissue representativity and information about the presence of lymphocytes can easily be obtained by making haematoxylin-eosin stains of the sections "before" and "after" the sections used for DNA extraction. It is recommended that these slides are evaluated by an experienced haematopathologist, who also has the immunostains of the cases available. Use of this information in the evaluation of the molecular data clearly benefits the interpretation of clonality results. A strictly quantitative approach using the criteria based on ratio determination, on the other hand, can easily miss a clonal process or can erroneously diagnose "clonality".

Still, small but predominant clonal-like peaks in a polyclonal background can be difficult to evaluate, especially when the sample has suboptimal DNA quality that prevents proper evaluation of all PCR targets. Duplicate analysis and look for consistency of the pattern, as well as sufficient DNA input per PCR and the correlation with the histology, will facilitate the evaluation of the case. Any tissue that has a limited number of $\mathrm{T}$ cells can be difficult to interpret as well. A dominant, seemingly clonal TCR rearrangement in such a case may represent a true clonal signal or pseudoclonality due to selective amplification from only a few target cells. In particular, TCRG gene rearrangement patterns can be complicated to make the distinction between clonal and polyclonal because of the restricted TCRG

\section{A: IGH tube B}
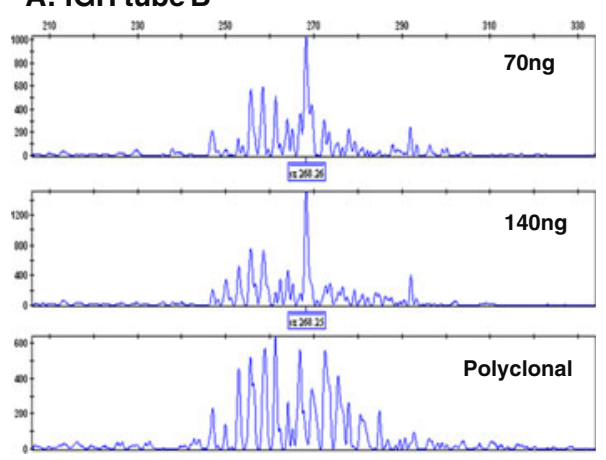

Fig. 4 DNA input determines the ratio between clonal product and polyclonal background. GeneScan rearrangement patterns are shown. Two different DNA concentrations of the same sample were used as input in the PCRs. A polyclonal control sample for each target is included. A clonal $I G H$ and a clonal $T C R G$ rearrangement within

\section{B: TCRG tube B}

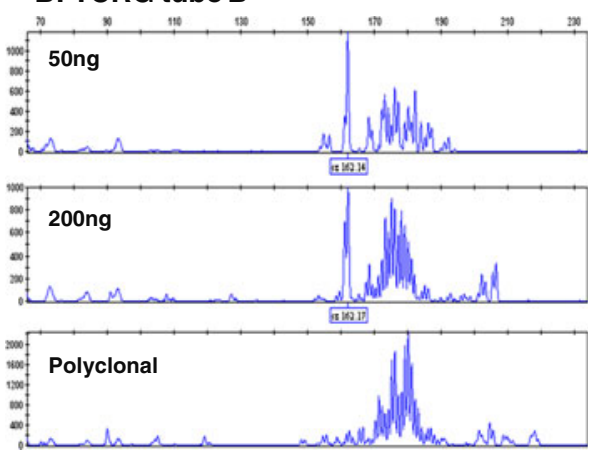

polyclonal signal can be observed in $\mathbf{a}$ and $\mathbf{b}$, respectively. The presence of the polyclonal background signals changes due to the altered DNA input per PCR. There is no severe effect on DNA input on the pattern when there is no polyclonal background signal present (c) 
germline repertoire and the limited junctional diversity. The multi-target approach combined with duplicate analysis is very helpful: The detection of clonal products that are consistently present in multiple PCR targets favours the presence of a true clone. We are aware that the TCRG PCR design with multiple size distributions with several fluorochromes can make the interpretation more difficult in some cases. It is of course helpful to see many cases and learn the "molecular morphology". In addition, we strongly recommend evaluation of the integrated (both fluorochromes combined) gene rearrangement patterns in one pattern above evaluation of the patterns of the individual fluorochromes because the evaluation of the integrated pattern decreases the likelihood of scoring seemingly dominant peaks as clonal.

Although interpretation of a molecular clonality result using the histopathological information in a way may be seen as "molecular morphology", there are strict criteria for interpretation of clonality: A clonal result should be detectable in duplicate either in the presence of hardly any polyclonal background or dominantly present in a Gaussian curve of polyclonal background signals, or within the expected size range that contains rare gene rearrangements (such as the $\mathrm{Vk} 2 / \mathrm{Vk} 4 / \mathrm{Vk} 5-\mathrm{JK} /$ - Kde rearrangements). A minority of the cases may still display gene rearrangement patterns that are difficult to judge as truly clonal, oligoclonal or polyclonal. However, duplicate assessment of the PCR targets combined with the performance of the multiple-target approach clearly assists in the evaluation. In fact, the consistency of the results in multiple PCR targets can be regarded as an internal control. In general in case of ambiguous results, we recommend to repeat the PCR, using the same DNA sample or a second independent DNA sample from the same or an independent representative tissue and check for consistency of results. In addition, verification with an alternative technique like Heteroduplex analysis may be helpful (see below).

GeneScanning and heteroduplex analysis as complementary readouts

GeneScanning is the most preferred readout for clonality analysis. The single-stranded PCR products are separated in a high-resolution gel or polymer according to their length only. It has a high resolution of 1-2 nt that allows distinction between a single clonal peak of particular size and a polyclonal pattern of gene rearrangements. In addition, GeneScanning can reach sensitivities of about $1 \%$ of clonal lymphocytes (although this level of sensitivity is not relevant in a pathology setting), and by being fully automated, it is less labor intensive and a rapid technique. However, GeneScanning requires expensive equipment. Chip-based microfluidic electrophoresis (Agilent) might be a good alternative for GeneScanning, as the instrument itself has a lower cost and the consumables are less expensive. Importantly, the currently available microfluidic chip-electrophoresis devices are not sufficiently sensitive to detect clonal products in a polyclonal background for $I G H$ PCR or complex $I G K$ rearrangements patterns [28]. In this respect, it is important to point out that conventional agarose electrophoresis by no means is suitable for clonality assessment because of its insufficient resolution.

Heteroduplex analysis uses double-stranded PCR products and takes advantage of the length and composition of the junctional regions. The technique has a detection limit of about $\sim 5-10 \%$ (a level entirely sufficient in a pathology setting), dependent on the PCR target and the system used, i.e. manual or commercial gels and DNA staining procedures. The detection limit of heteroduplex analysis is influenced by the frequency of polyclonal lymphocytes since the formation of heteroduplexes will also consume a part of the monoclonal PCR products [29]. Clearly, GeneScanning and heteroduplex analysis have complementary value. Evaluation of $I G K$ gene rearrangement patterns by GeneScan can be difficult, which is related to the limited junctional regions in $I G K$. In addition, the rearrangement patterns are represented as small Gaussian curves, based on the PCR design with the different $I G K$ primers at scattered positions towards the recombination signal sequences, in order to have minimal cross-annealing to other VK family gene segments [1]. By exploiting both size and composition, heteroduplex analysis is particularly useful when a "clonal" product has the same amplicon length as the predominant $I G K$ polyclonal gene rearrangements in GeneScan (Fig. 5a, d and e). Furthermore, heteroduplex analysis is useful to detect clonal products outside the size range assessed by Genescanning (Fig. 5b, f). On other hand, heteroduplex analysis is less sensitive compared to GeneScanning, so reproducible clonal peaks with low signal intensity cannot be detected by heteroduplex analysis (Fig. 5c, g).

Recognition of non-specific products in the rearrangement patterns

Besides the knowledge of technical information such as DNA quality and DNA input, also sufficient knowledge of the gene loci, the PCR design and the non-specific products is of utmost importance in the final evaluation of clonality testing. Since the BIOMED-2 PCRs are multi-plex PCRs, non-specific amplification of products may occur in PCRs and are documented ([1] and an updated table can be found at http://www.euroclonality.org under the Frequently Asked Questions). The use of a polyclonal sample in each run is advised not only to confirm that both the primers and the PCR work well but also to see non-specific products. An example is the dominant product of $345 \mathrm{bp}$ in the $I G H$-DJ tube A, which results from cross-annealing from an upstream 

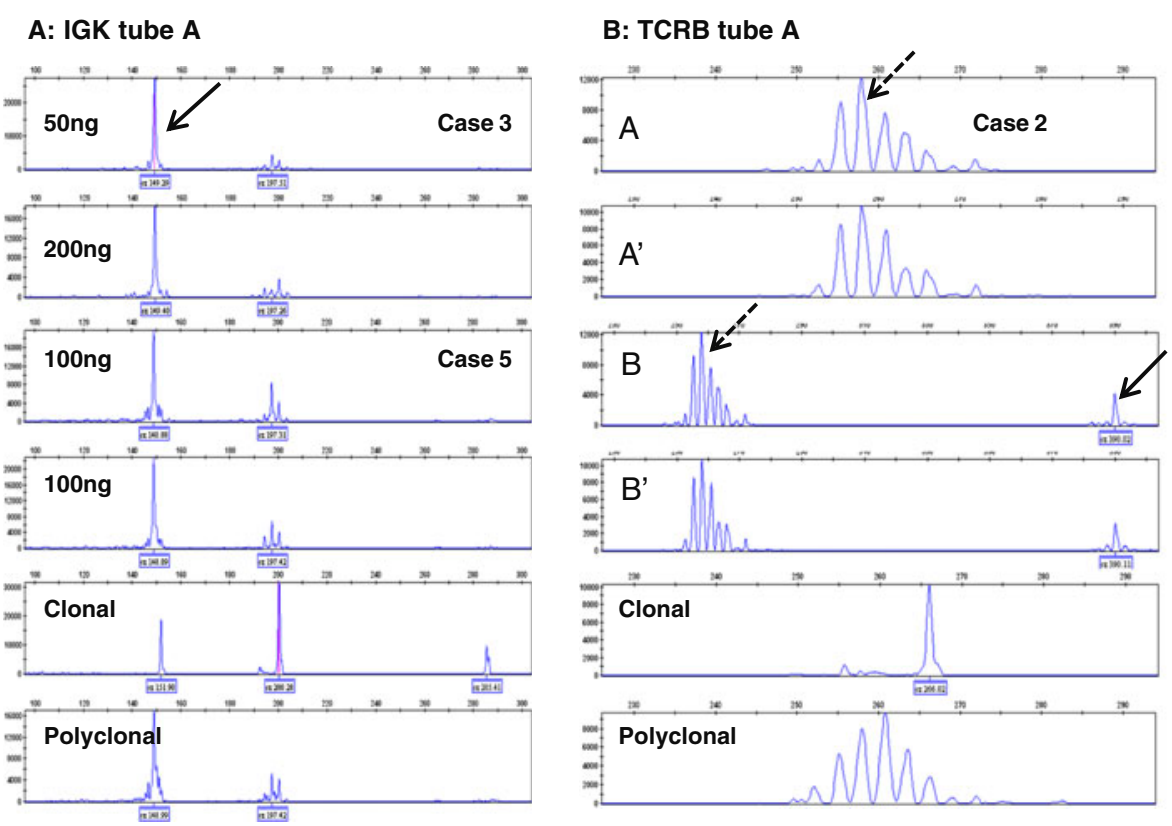

\section{C: TCRG tube A}
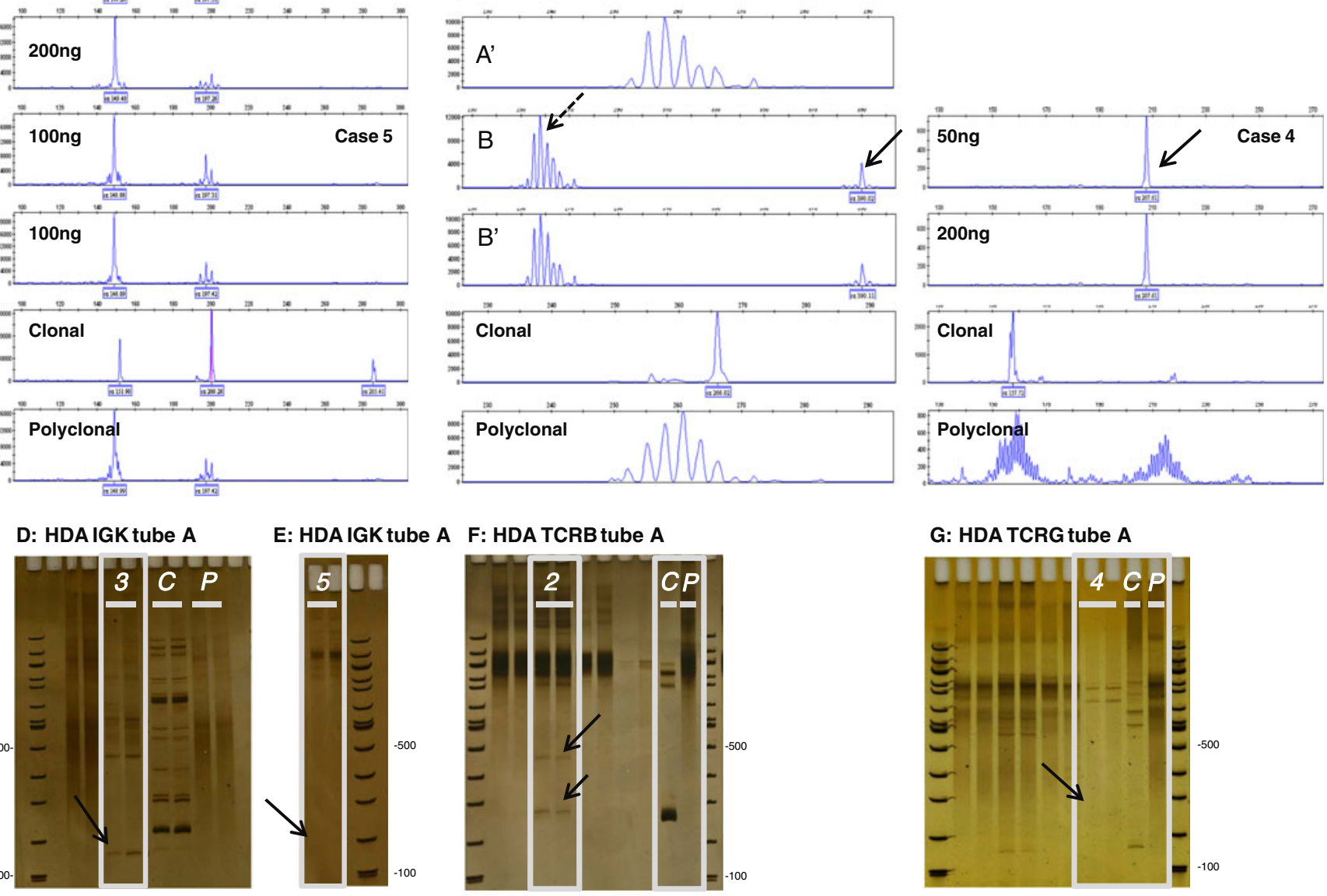

Fig. 5 Heteroduplex and Genescanning yield complementary information. $I G K$-VJ (tube A) gene rearrangement patterns showing monoclonality of case 3 in GeneScan (a) and heteroduplex analysis (d), and polyclonality of case 5 in GeneScan (a) and heteroduplex analysis (e). Heteroduplex analysis shows clonal TCRB-products (tube A) in case 2 (f, two bands indicated), which cannot be easily detected in the GeneScan using the normal size range although a strange peak can be seen ( $\mathrm{A}$ and $\mathrm{A}$

$\mathrm{DH} 2$ to $\mathrm{J}$ (germ line) and is present in the PCRs except when there is a true clonal rearrangement. The primers are then being consumed by the clonal rearrangement, and as a result, the non-specific peak disappears. Other predominant, nonspecific peaks may arise in the context of the sample. When there are low numbers of target $\mathrm{T}$ lymphocytes in the sample, a non-specific peak at $273 \mathrm{bp}$ in the TCRB tube A may come up. Non-specific products of 229 and $211 \mathrm{bp}$ are often seen in the IGH-FR2 and FR3 PCRs, respectively. Apart from awareness of the non-specific products, also knowledge about the gene loci and the design of the PCR is important for evaluation of clonality. The gene loci of $I G K$ and TCRB can have multiple gene rearrangements at a single allele, which may complicate evaluation. This issue is specifically addressed by Langerak and Van Dongen [30].

" in $\mathbf{b}$, peaks indicated by dashed arrow). Widening the size range however (B and $\mathrm{B}^{\prime \prime}$ in $\mathbf{b}$ ) clearly shows the clonal peak. The $T C R G$-clonal product (tube A) is clonal on GeneScan (c) but the intensity is too low to allow detection on heteroduplex analysis $(\mathbf{g})$. Heteroduplex analysis was done using commercial, ready-to-use polyacrylamide gels (GeneGel Excell 12,5/24 Kit, GE Healthcare) and duplexes were visualized by silver staining

Recognition of aberrant rearrangements: TCRG J-J rearrangement as an example

The majority $(95 \%)$ of the rearrangements fall within the size range provided by the BIOMED-2 group. However, a very minority of truly clonal rearrangements may fall just outside the size range. In addition, there are clonal rearrangements that are clearly undersized or oversized [31-33]. To make sure that these aberrantly sized rearrangements may not go unnoticed, it is advised to examine a broad size window for rearrangement detection. A critical view of convincingly dominant products that fall in the size range of known rare rearrangements, such as the TCRG-V11 rearrangement, is recommended, too. Recently, we identified an aberrant $T C R G$ rearrangement in two unrelated 


\section{a}

\section{TCRG tube B}

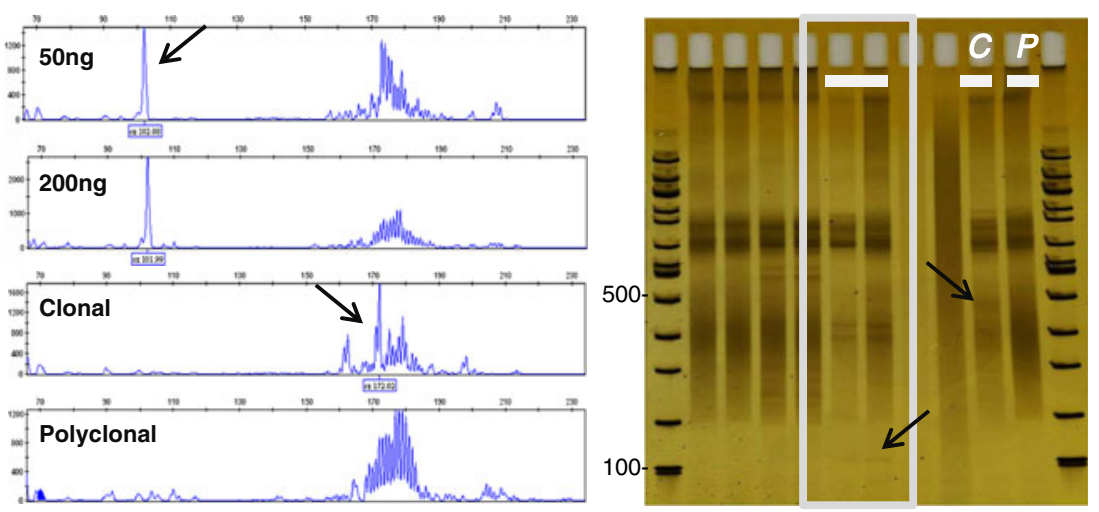

b

TCRG 7p14

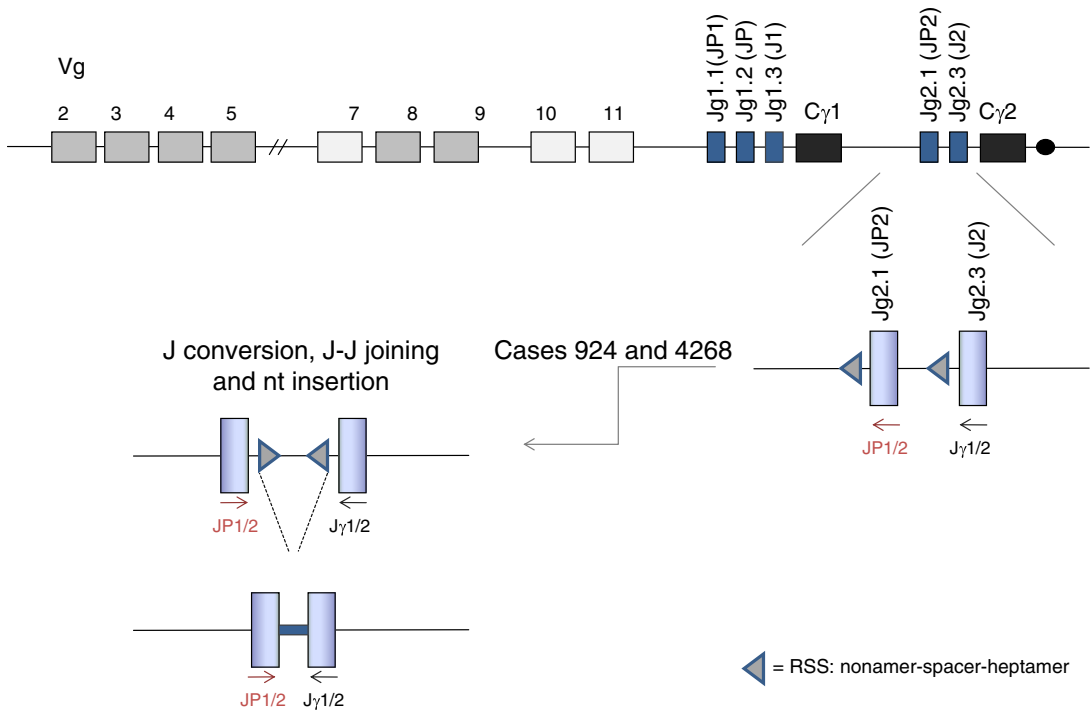

\section{C}

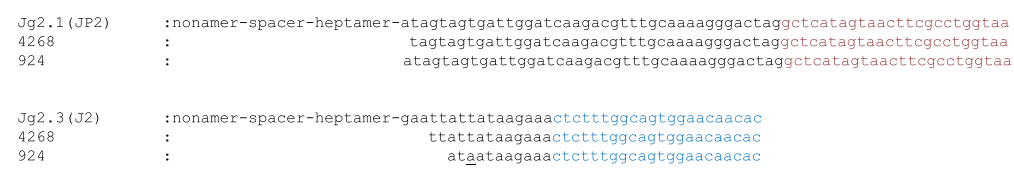

PCR product 924 : ttaccaggcgaagttactatgagcctagtcccttttgcaaacgtcttgatccaatcactactat- $(24 \mathrm{nt})$-atäataagaaactctttggcagtggaacaacac

Fig. 6 An aberrant TCRG-JJ rearrangement detected. a Both the GeneScan pattern and the heteroduplex gel show a weak "clonal" product within the $\mathrm{V} \gamma 11$ size range. Schematic overview (b) and sequence information (c) of the aberrant TCRG-JJ rearrangement 
cases, one case was a B cell lymphoma with a suspicious $\mathrm{T}$ cell infiltrate, and the other case was a duodenum biopsy of a patient with refractory coeliac disease. Both rearrangements were small in size (110-124 nt in size) and came up in $T C R G-\mathrm{V} 11$ size range of the TCRG tube B pattern. Because TCRGV11 rearrangements are rare, the dominant products were analysed by sequencing. Both cases displayed aberrant inversional J $\gamma 2$.1(JP2)-J $\gamma 2.3(\mathrm{~J} 2)$ rearrangements (Fig. 6), excluding strange mispriming of the primers. Although this is not a functional TCR VJ rearrangement that results into a functional TCR $\gamma$ protein chain, it is a clonal gene rearrangement, similar to clonal TCRB-DJ, IGH-DJ and $I G K$-DE gene rearrangements. The question of course is how to report this rearrangement, especially since both these $T C R G$-JJ rearrangements were detected as isolated rearrangements. One may decide not to report the inversional TCRG-JJ rearrangement, based on the concept that this might be a germ-line rearrangement. However, the polyclonal background signal detected in the patterns would argue against a germ-line event. In our opinion, it may be the best to choose to report the aberrant $T C R G$-JJ rearrangement as a non-functional clonal rearrangement of unknown significance and recommend follow-up of the patient.

\section{Concluding remarks}

A good practical workflow including replicate testing and critical assessment of the obtained gene rearrangement patterns is of utmost importance for proper evaluation of clonality testing. Based on the discussions we had in the EuroClonality workshops, "Clonality Assessment in Pathology", these aspects, as well as knowledge on pitfalls, on PCR design and the evaluation of clonality patterns can avoid misinterpretation of the data. The issues addressed will help to overcome problems that can be encountered in the routine diagnostic setting. The true meaning of clonality is also dependent on the histological and clinical context. It is therefore recommended to integrate the molecular data with those from pathology for appropriate interpretation to reach the most reliable diagnosis.

\section{References}

1. van Dongen JJ, Langerak AW, Brüggemann M et al (2003) Design and standardization of PCR primers and protocols for detection of clonal immunoglobulin and T-cell receptor gene recombinations in suspect lymphoproliferations: report of the BIOMED-2 Concerted Action BMH4-CT98-3936. Leukemia 17:2257-2317

2. Langerak AW, Groenen PJTA, van Krieken JHJM, van Dongen JJM (2007) Immunoglobulin/T-cell receptor clonality diagnostics. Expert Opin Med Diag 1:451-461

3. Groenen PJ, Langerak AW, van Dongen JJ, van Krieken JH (2008) Pitfalls in TCR gene clonality testing: teaching cases. J Hematop 1:97-109
4. Evans PA, Pott CH, Groenen PJ et al (2007) Significantly improved PCR-based clonality testing in B-cell malignancies by use of multiple immunoglobulin gene targets. Report of the BIOMED-2 Concerted Action BHM4-CT98-3936. Leukemia 21:207-214

5. Brüggemann M, White H, Gaulard P et al (2007) Powerful strategy for polymerase chain reaction-based clonality assessment in T-cell malignancies. Report of the BIOMED-2 Concerted Action BHM4 CT98-3936. Leukemia 21:215-221

6. Schraders M, de Jong D, Kluin P, Groenen P, van Krieken H (2005) Lack of Bcl-2 expression in follicular lymphoma may be caused by mutations in the BCL2 gene or by absence of the $t$ $(14 ; 18)$ translocation. J Pathol 205:329-335

7. Langerak AW, Molina TJ, Lavender FL, Pearson D, Flohr T, Sambade C, Schuuring E, Al Saati T, van Dongen JJ, van Krieken JH (2007) Polymerase chain reaction-based clonality testing in tissue samples with reactive lymphoproliferations: usefulness and pitfalls. A report of the BIOMED-2 Concerted Action BMH4CT98-3936. Leukemia 21:222-229

8. McClure RF, Kaur P, Pagel E, Quillette PD, Hotegaard CE, Treptow $\mathrm{CL}$ et al (2006) Validation of immunoglobulin gene rearrangement detection by PCR using commercially available BIOMED-2 primers. Leukemia 20:176-179

9. Chute DJ, Cousar JB, Mahadevan MS, Siegrist KA, Silverman LM, Stoler MH (2008) Detection of immunoglobulin heavy chain gene rearrangements in classic Hodgkin lymphoma using commercially available BIOMED-2 primers. Diagn Mol Pathol 17:65-72

10. Hebeda KM, Van Altena MC, Rombout P, Van Krieken JH, Groenen PJ (2009) PCR clonality detection in Hodgkin lymphoma. J Hematop 2:34-41

11. Burack WR, Laughlin TS, Friedberg JW, Spence JM, Rothberg PG (2010) PCR assays detect B-lymphocyte clonality in formalinfixed, paraffin-embedded specimens of classical Hodgkin lymphoma without microdissection. Am J Clin Pathol 134:104-111

12. Matthews C, Catherwood M, Morris TC, Alexander HD (2004) Routine analysis of IgVH mutational status in CLL patients using BIOMED-2 standardized primers and protocols. Leuk Lymphoma 45:1899-1904

13. Lassmann S, Gerlach UV, Technau-Ihling K, Werner M, Fisch $P$ (2005) Application of BIOMED-2 primers in fixed and decalcified bone marrow biopsies: analysis of immunoglobulin $\mathrm{H}$ receptor rearrangements in B-cell non-Hodgkin's lymphomas. J Mol Diagn 7:582-591

14. Liu H, Bench AJ, Bacon CM, Payne K, Huang Y, Scott MA, Erber WN, Grant JW, Du MQ (2007) A practical strategy for the routine use of BIOMED-2 PCR assays for detection of B- and T-cell clonality in diagnostic haematopathology. Br J Haematol 138:31-43

15. Tan BT, Seo K, Warnke RA, Arber DA (2008) The frequency of immunoglobulin heavy chain gene and T-cell receptor gammachain gene rearrangements and Epstein-Barr virus in ALK+ and ALK - anaplastic large cell lymphoma and other peripheral T-cell lymphomas. J Mol Diagn 10:502-512

16. Chen YL, Su IJ, Cheng HY, Chang KC, Lu CC, Chow NH, Ho CL, Huang W (2010) BIOMED-2 protocols to detect clonal immunoglobulin and T-cell receptor gene rearrangements in B- and T-cell lymphomas in southern Taiwan. Leuk Lymphoma 51:650-655

17. Ibrahim HA, Menasce LP, Pomplun S, Burke M, Bower M, Naresh KN (2011) Presence of monoclonal T-cell populations in B-cell posttransplant lymphoproliferative disorders. Mod Pathol 24:232-240

18. Zaki MA, Wada N, Kohara M, Ikeda J, Hori Y, Fujita S, Ogawa H, Sugiyama H, Hino M, Kanakura Y, Morii E, Aozasa K (2011) Presence of B-cell clones in T-cell lymphoma. Eur J Haematol 86:412-419

19. Groenen PJ, Blokx WA, Diepenbroek C, Burgers L, Visinoni F, Wesseling P, van Krieken JH (2011) Preparing pathology for personalized medicine: possibilities for improvement of the preanalytical phase. Histopathology 59(1):1-7 
20. Lenze D, Müller HH, Hummel MJ (2012) Considerations for the use of formalin-fixed and paraffin-embedded tissue specimens for clonality analysis. Hematopath (in press)

21. Halldorsdottir AM, Zehnbauer BA, Burack WR (2007) Application of BIOMED-2 clonality assays to formalin-fixed paraffin embedded follicular lymphoma specimens: superior performance of the IGK assays compared to IGH for suboptimal specimens. Leuk Lymphoma 48:1338-1343

22. Fend F, Tzankov A, Bink K, Seidl S, Quintanilla-Martinez L, Kremer M, Dirnhofer S (2008) Modern techniques for the diagnostic evaluation of the trephine bone marrow biopsy: methodological aspects and applications. Prog Histochem Cytochem 42:203-252

23. Talaulikar D, Shadbolt B, McNiven M, Dahlstrom JE (2008) DNA amplification from formalin-fixed decalcified paraffin-embedded bone marrow trephine specimens: does the duration of storage matter? Pathology 40:702-706

24. Zanssen S (2004) Single cell PCR from archival stained bone marrow slides: a method for molecular diagnosis and characterization. J Clin Lab Anal 18:176-181

25. Thurber SE, Zhang B, Kim YH, Schrijver I, Zehnder J, Kohler S (2007) T-cell clonality analysis in biopsy specimens from two different skin sites shows high specificity in the diagnosis of patients with suggested mycosis fungoides. J Am Acad Dermatol 57:782-790

26. Dabiri S, Morales A, Ma L, Sundram U, Kim YH, Arber DA, Kim J (2011) The frequency of dual TCR-PCR clonality in granulomatous disorders. J Cutan Pathol 38:704-709
27. Zhang B, Beck AH, Taube JM, Kohler S, Seo K, Zwerner J, Viakhereva N, Sundram U, Kim YH, Schrijver I, Arber DA, Zehnder JL (2010) Combined use of PCR-based TCRG and TCRB clonality tests on paraffin-embedded skin tissue in the differential diagnosis of mycosis fungoides and inflammatory dermatoses. $\mathrm{J}$ Mol Diagn 12:320-327

28. Zamò A, Bertolaso A, van Raaij AWM, Mancini F, Scardoni M, Montresor M, Menestrina F, van Krieken JHJM, Chilosi M, Groenen PJTA, Scarpa A (2012) Application of microfluidic technology to the BIOMED-2 protocol for detection of B-cell clonality. J Mol Diagn 14:30-37

29. Langerak AW, Szczepanski T, van der Burg M, Wolvers-Tettero ILM, van Dongen JJM (1997) Heteroduplex PCR analysis of rearranged $\mathrm{T}$ cell receptor genes for clonality assessment in suspect T cell proliferations. Leukemia 11:2192-2199

30. Langerak AW, van Dongen JJM (2012) Multiple clonal Ig/TCR products: implications for interpretation of clonality findings. $\mathrm{J}$ Hematopath (in press)

31. Vargas RL, Felgar RE, Rothberg PG (2008) Detection of clonality in lymphoproliferations using PCR of the antigen receptor genes: does size matter? Leuk Res 32:335-338

32. Langerak AW (2008) Undersized, oversized? It is not one-size-fits-all in lymphoid clonality detection. Leukemia Res 322:203-204

33. Rothberg PG, Langerak AW, Verhaaf B, van Dongen JJM, Burack WR, Johnson MD, Laughlin TS, Payne K, Figueiredo L, Bandoh B, Yan Q, Bacon CM, Wrigh P, Bench A, Du M-Q, Liu H (2012) Does size matter? Clonal antigen receptor gene PCR products outside of the expected size range. J Hematopath (in press) 